\title{
PREPARE: presurgery physiotherapy for patients with degenerative lumbar spine disorder: a randomized controlled trial
}

Yvonne Lindbäck, Hans Tropp, Paul Enthoven, Allan Abbott and Birgitta Öberg

The self-archived postprint version of this journal article is available at Linköping University Institutional Repository (DiVA):

http:/ / urn.kb.se/ resolve?urn=urn:nbn:se:liu:diva- 147620

N.B.: When citing this work, cite the original publication.

Lindbäck, Y., Tropp, H., Enthoven, P., Abbott, A., Öberg, B., (2017), PREPARE: presurgery

physiotherapy for patients with degenerative lumbar spine disorder: a randomized controlled trial, The spine journal. https:// doi.org/ 10.1016/j.spinee.2017.12.009

Original publication available at:

https:// doi.org/ 10.1016/j.spinee.2017.12.009

Copyright: Elsevier

http:// www.elsevier.com/

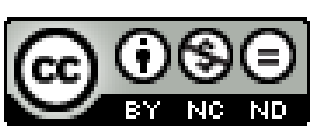




\section{Accepted Manuscript}

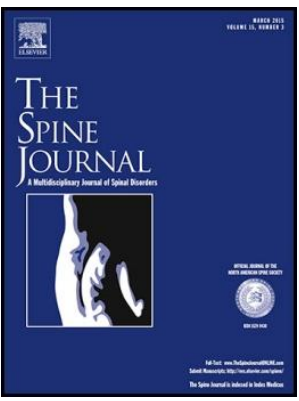

Title: Prepare: pre-surgery physiotherapy for patients with degenerative lumbar spine disorder: a randomized controlled trial

Author: Yvonne Lindbäck, Hans Tropp, Paul Enthoven, Allan Abbott, Birgitta Öberg

PII:

S1529-9430(17)31217-2

DOI:

https://doi.org/10.1016/j.spinee.2017.12.009

Reference:

SPINEE 57561

To appear in: The Spine Journal

Received date: $9-8-2017$

Revised date: 25-10-2017

Accepted date: 11-12-2017

Please cite this article as: Yvonne Lindbäck, Hans Tropp, Paul Enthoven, Allan Abbott, Birgitta Öberg, Prepare: pre-surgery physiotherapy for patients with degenerative lumbar spine disorder: a randomized controlled trial, The Spine Journal (2017), https://doi.org/10.1016/j.spinee.2017.12.009.

This is a PDF file of an unedited manuscript that has been accepted for publication. As a service to our customers we are providing this early version of the manuscript. The manuscript will undergo copyediting, typesetting, and review of the resulting proof before it is published in its final form. Please note that during the production process errors may be discovered which could affect the content, and all legal disclaimers that apply to the journal pertain. 
PREPARE: Pre-surgery physiotherapy for patients with degenerative lumbar spine disorder: a randomized controlled trial

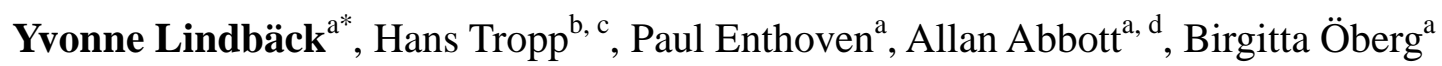

Yvonne Lindbäck* - yvonne.lindback@liu.se; Hans Tropp - hans.tropp@ regionostergotland.se;

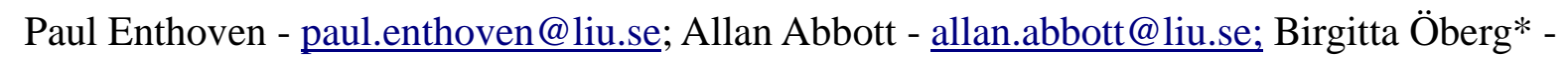

\section{birgitta.oberg@liu.se}

${ }^{a}$ Department of Medical and Health Sciences, Division of Physiotherapy, Faculty of Medicine and Health Sciences, Linköping University, Linköping, Sweden.

${ }^{\mathrm{b}}$ Department of Spinal Surgery and Department of Clinical and Experimental Medicine, Linköping University, Linköping, Sweden.

${ }^{c}$ Center for Medical Image Science and Visualization (CMIV), Linköping University, Linköping, Sweden.

${ }^{\mathrm{d}}$ Faculty of Health Science and Medicine, Bond University, Gold Coast, Queensland, 4229, Australia

*Corresponding author. Mailing address: Dep of Medical and Health Sciences, Div of Physiotherapy, Linköping University, SE-581 83 Linköping, Sweden telephone number +46 13284743, e-mail address yvonne.lindback@liu.se Institutional URL: www.imh.liu.se/fysioterapi

Trial registration: ClinicalTrials.gov reference: NCT02454400 (Trial registration date: August 31st 2015).

\section{Abstract}

Background Context - Surgery due to disc herniation, or spinal stenosis results mostly in large improvement in the short-term, but mild to moderate improvements for pain and disability at long-term follow-up. Prehabilitation has been defined as augmenting functional capacity prior to surgery, which may have beneficial effect on outcome after surgery. 
1 Purpose - The aim was to study if pre-surgery physiotherapy improves function, pain and health in

2 patients with degenerative lumbar spine disorder scheduled for surgery.

3 Study Design - A single blinded, 2-arm, RCT.

4 Patient Sample - 197 patients were consecutively included at a Spine Clinic. The inclusion

5 criteria were; patients scheduled for surgery due to disc herniation, spinal stenosis,

6 spondylolisthesis or DDD, 25 to 80 years of age.

7 Outcome Measures - Primary outcome was Oswestry Disability Index (ODI). Secondary

8 outcomes were pain intensity, anxiety, depression, self-efficacy, fear avoidance, physical activity

9 and treatment effect.

10 Methods - Patients were randomized to either - pre-surgery physiotherapy or standardized

11 information, with follow-up after the pre-surgery intervention as well as 3 and 12 months post-

12 surgery. The study was funded by regional research funds for US $\$ 77,342$. No conflict of interest

13 is declared.

14 Results - The pre-surgery physiotherapy group had better ODI, VAS back pain, EQ-5D, EQ-

15 VAS, FABQ-PA, SES and HADS depression scores and activity level compared to the waiting-

16 list group after the pre-surgery intervention. The improvements were small, but larger than the

17 study specific minimal clinical important change (MCIC) in VAS back and leg pain, EQ-5D and

18 FABQ-PA and almost in line with MCIC in ODI and PCS in the physiotherapy-group. Post-

19 surgery difference between groups only maintained for higher activity level in the physiotherapy-

20 group.

21 Conclusions - Pre-surgery physiotherapy decreases pain, risk for avoidance behavior and

22 worsening of psychological well-being and improves quality of life, and physical activity levels

23 prior to surgery compared to waiting-list controls. These results were only maintained for

24 activity levels post-surgery. Still, pre-surgery selection, content, dosage of exercises and

25 importance of being active in a pre-surgery physiotherapy intervention is of interest to study

26 further to improve long-term outcome. 
1

2 Spinal stenosis is the most common degenerative lumbar spine disorder requiring surgery [1, 2],

3 followed by disc herniation and to a smaller extent, spondylolisthesis and degenerative disc

4 disease (DDD) [2]. Surgery for radiculopathy due to disc herniation, and spinal stenosis has been

5 shown to result in large improvements at 3 months follow-up, but mild to moderate

6 improvements for pain and disability at 5-year follow-up [3, 4]. Guideline recommendations are that non-surgical interventions should be exhausted prior to decision making about surgery for spinal stenosis [5], disc herniation [6] and spondylolisthesis [7]. However, only $10 \%$ of clinical studies investigating the effect of surgery have information about non-surgical intervention prior to surgery for spinal stenosis [8]. Prehabilitation has been defined as augmenting functional capacity prior to surgery [9], which may have beneficial effect on outcome after surgery [10]. Randomized controlled trials (RCT) suggest that pre-surgery physiotherapy as well as pain education improves outcomes in function and health behaviour in patients with radiculopathy $[11,12]$. Apart from these interventions, guidelines for chronic low back pain (LBP) recommended use of tailor-made supervised exercise program and behavioural approach. A stratified pre-surgery physiotherapy, tailor-made supervised exercise program and use of behavioural approaches possibly influencing biological-, psychological- and social components, has not previously been evaluated in patients with degenerative spinal disorder scheduled for surgery. The aim was to study if pre-surgery physiotherapy improves function, pain and health in patients with degenerative lumbar spine disorder scheduled for surgery.

\section{Methods}

\section{Study design}

This is a single blinded, 2-arm, RCT. The primary end-point at 1-year follow-up is presented here together with follow-up after pre-surgery intervention and at 3-month post-surgery. The trial received ethical approval from the Regional Ethics Committee (2012/167-31). The trial protocol 
1 has been published on (ClinicalTrials.gov XX=excluded in blinded manuscript, available in a

2 separate document in the submission). We follow the CONSORT guidelines for RCT.

\section{$3 \quad$ Participants and setting}

4 All patients were referred to a Spine Clinic at a University Hospital in XX and consecutively 5 included October 2012 to March 2015. The inclusion criteria were; patients aged 25-80 years

6 scheduled for surgery for degenerative lumbar spine disorder; presence of LBP and/or leg pain

7 due to disc herniation, spinal stenosis, spondylolisthesis (Grade 1-2), DDD; diagnosis confirmed

8 by magnetic resonance imaging; pain level high enough to indicate surgical intervention; fluency

9 in Swedish. Exclusion criteria: Patients that were in need of acute surgery or re-surgery on the

10 same level; had severe spinal pathology (such as osteoporosis or fusion > 4 levels) or other

11 severe diagnoses.

12 Intervention

13 Waiting-list group

14 Patients received standardized information about surgery from an orthopedic surgeon, post-

15 surgery rehabilitation and advice to stay active.

16 Physiotherapy group

17 Patients received pre-surgery physiotherapy intervention twice a week for 9 weeks. The program

18 included:

19 1. Physiotherapy according to a treatment based classification (TBC) [13]; a) Specific exercises

20 and mobilization, or b) Motor control exercises or c) Traction.

21 2. Tailor-made general supervised exercise program.

22 3. Behavioral approach to reduce fear avoidance and increase activity level.

23 Patients received the same standardized information as the waiting-list group. The physiotherapy

24 intervention was performed at one of eleven physiotherapy clinics in the County Council. For

25 each patient the physiotherapist followed a checklist with content and progression planned for

26 each treatment-session, manual techniques and exercise was tailor-made. The post-surgery

27 rehabilitation included feedback on posture and walking, a home exercise-program, and daily 
1 walking, which was followed-up and progressed after 6 weeks. This together with intervention

2 for the waiting-list group and the spinal surgery followed clinical routine [14]. Details about the

3 intervention and TBC are described in the study-protocol [15].

\section{Outcome}

5 Questionnaire based patient reported outcome measures (PROMs) were collected at baseline,

6 after 9 weeks intervention pre-surgery, 3 months and 1-year post-surgery.

$7 \quad$ Primary outcome measure

8 Function and activity limitation was measured by Oswestry Disability Index (ODI) [16].

$9 \quad$ Secondary outcome measures

10 Pain was evaluated with Visual Analogue Scale (VAS) [17] and pain duration. Health related

11 quality of life with; SF-36 [18] and EQ-5D [19]. Anxiety and depression with; the Hospital

12 Anxiety and Depression Scale (HADS) [20] and self-efficacy with; Self-Efficacy Scale (SES)

13 [21]. Fear avoidance was measured by Fear Avoidance Belief Questionnaire - Physical Activity

14 (FABQ-PA) [22]. Treatment effect was measured by Patient Global Impression of Change

15 (PGIC) [23]. Physical activity level was measured by a question with five answer options. Study-

16 protocol provides further information about the PROMs [15].

\section{Sample size}

18 Based on a Minimally Clinical Important Change (MCIC) of 10 percent on the ODI [24], a

19 standard deviation for the ODI $=20$, a significance level of $\mathrm{p}=0.05$, a power of $80 \%$, an estimated

2064 patients were required in each of the intervention groups.

\section{Randomization}

Block randomization was used. For each randomization block, sealed opaque envelopes were prepared with a 1:1 ratio of allocation to the two groups. After baseline measurement, an independent physiotherapist informed the patient about group allocation.

\section{Blinding}

26 The physiotherapists performing the physical examination was blinded to the randomization,

27 while patients and the treating physiotherapist were not. 


\section{1}

2

3

\section{Statistical methods}

For between-group comparisons of demographic data, the unpaired Student's $t$-test or Mann-

Whitney $U$ test for continues variables and the Chi-Square test for categorical variables were used. Two way ANCOVA and repeated measures (covariates = age, gender and diagnosis) were used for comparison over time. ANCOVA was adjusted for baseline values for each PROMs. Cohen's $d$ effect sizes were also calculated for changes in groups over time, whereas Cohen's $d=$ 0.20 is considered a small, $d=0.50$ is a medium and $d=0.80$-infinity is a large effect size [25].

For Chi-Square test with 4 degrees of freedom, Cramer's $\mathrm{V}=0.05$ is considered a small, $\mathrm{V}=$ 0.15 is a medium and $\mathrm{V}=0.25$ is considered a large effect size in the analysis of activity level in the two groups [25].

Data collected at different time points was analysed according to the Intention To Treat (ITT) principle. Missing data in PROMs, except for physical activity level and PGIC, were replaced through multiple imputation, based on group data from baseline and actual time point. Multiple imputation by chained equations (MICE) procedure named fully conditional specification method (FCS) in SPSS with ten data sets was used [26]. Constrains were applied for each variable according to the range of each scale. The mean of ten imputation sets was used for each of the PROMs. A sensitivity analysis comparing Per Protocol (PP) data with ITT data [27] showed no significant differences. The study specific MCIC was calculated by Minimal Detectible Change (MDC) and Optimal Cutoff Point (OCP) for PROMs with change within group after the pre-surgery intervention [28]. MDC was calculated by; $1.96 * \sqrt{2} * \mathrm{SEM}$.

Further, PGIC was dichotomised into "improved" (= very much better, much better or slightly better) and "no change" and was used for the sensitivity and specificity for the receiver operating characteristic (ROC) curve. The OCP was defined by the sensitivity and specificity to reach lowest percentage of misclassified [28]. An Area Under the Curve (AUC) $\geq 0.70$ depicts a satisfactory accuracy for the model [29]. Statistical analysis was performed using IBM SPSS statistics version 23. The level of significance was set to 0.05 . 


\section{Results}

2 In total 197 patients with degenerative lumbar spine disorder were included. The mean (standard

3 deviation (SD)) age was $59(12.5)$ years and $105(53 \%)$ were women. Ninety-one patients (51\%)

4 had back pain for > 2 years and $63(35 \%)$ leg pain for > 2 years. In the last 12 months 115 patients

$5 \quad(58 \%)$ had $\geq 1$ visit at a physiotherapist or other caregiver, whereas $48(42 \%)$ reported

6 improvements, $52(46 \%)$ unchanged and $14(12 \%)$ worsening. They were randomized to either the

7 physiotherapy group $(n=99)$ or waiting-list group $(n=98)$ (Fig. 1). There were no significant

8 differences between the groups in baseline characteristics, except in PCS (Table 1, 2). Neither

9 were there significant differences in type of surgery (having fusion procedure or not).

10 One hundred sixty eight patients completed the questionnaire after the pre-surgery intervention.

11 There were no differences between the groups regarding the characteristics of patients who

12 dropped out, except for a larger proportion of patients in the dropout group had disk herniation, $\mathrm{n}$

$13=13(45 \%)$ versus $\mathrm{n}=27(16 \%, P=0.002)$.

14 On average, the patients in the physiotherapy group had 11 treatment sessions, with 43 (43\%) not 15 completing $\geq 12$ treatment-session quota for optimal adherence to treatment (Fig. 1). No adverse 16 events were reported.

Fig. 1, somewhere here

Fig. 1. CONSORT flow chart of the randomized controlled trial.

Table 1, somewhere here

Table 2, somewhere here

After the pre-surgery intervention (prior to surgery), the physiotherapy-group was significantly improved in ODI, VAS back and leg pain, EQ-5D, EQ-VAS and PCS compared to baseline. The waiting-list group was significantly improved in VAS leg pain and had deteriorated in 
1 (prior to surgery) showed significant differences in; ODI, VAS back pain, EQ-5D, EQ-VAS,

2 FABQ-PA, SES HADS depression, with advantage for the physiotherapy-group. Both groups

3 were improved in these PROMs at the two post-surgery time points, but without significant

4 differences between the groups (Table 3).

Table 3 somewhere here

The changes in the physiotherapy-group were larger than MDC in ODI, VAS back and leg, EQ-

5D, EQ-VAS, SF36 PCS and FABQ-PA and larger than OCP in VAS back and leg, EQ-5D, EQ-

VAS, and FABQ-PA. The deterioration in the waiting-list group was larger than MDC in HADS depression, smaller than MDC in SES and both HADS depression and SES were smaller than OCP. In four of the PROMs in the physiotherapy-group with significant improvements after presurgery intervention, AUC was $\geq 0.7$ for ODI, VAS leg pain, FABQ-PA and PCS (Table 4).

Table 4 somewhere here

In the physiotherapy-group there was a larger amount that reported higher physical activity after the pre-surgery intervention than in the waiting-list group $(P<0.001)$ with a large Cramer's V effect size of $0.391[25]$. The difference between the groups remained at 1-year follow-up $(P=$ 0.020), with a large Cramer's V effect size of 0.26 [25].

The physiotherapy-group had a larger amount reporting improvements than the waiting-list group in PGIC at the time point after the pre-surgery intervention (prior to surgery) $(P<0.001)$.

24 In the physiotherapy-group, $49 \%$ reported, "improved", compared to $17 \%$ in the waiting-listgroup. Thirteen percent reported "worse" in the physiotherapy-group compared to $42 \%$ in the waiting-list group (Fig. 2). There were no differences between the groups at 3 months- and at 1year follow-up. 
Fig. 2, somewhere here

3 Fig. 2. Patient Global Impression of Change after pre-surgery intervention for the physiotherapy$(\mathrm{n}=99)$ and waiting-list group $(\mathrm{n}=98)$. Original data.

\section{Discussion}

The study showed a more beneficial outcome in most PROMs for the physiotherapy-group compared to the waiting-list group after pre-surgery intervention. At post-surgery follow-ups, these differences had levelled out, except for a maintenance of higher physical activity level in the physiotherapy-group 1-year post-surgery. Most PROMs were improved with a small to moderate effect-size in the physiotherapy-group and two PROMs had deteriorations in the waiting-list group prior to surgery. The statistically significant improvements in ODI and VAS back and leg pain, EQ-5D, EQ-VAS, PCS and FABQ-PA in the physiotherapy-group after the pre-surgery intervention were small in relation to MCIC reported in previous literature [24]. According to the study specific MCIC, the improvements in the physiotherapy-group were larger than MDC in ODI, VAS back and leg pain, EQ-5D, EQ-VAS, SF-36 PCS and FABQ-PA and larger than OCP in VAS back and leg pain, EQ-VAS and FABQ-PA and almost in line with MCIC in ODI and PCS. This illustrates that these small changes are of importance in this patient-group.

21 The physiotherapy-group had improvements in FABQ-PA, while the waiting-list group had deterioration in HADS depression and SES, in both within- and between-groups comparison after the pre-surgery intervention. Commonly the waiting-list period is $2-5$ months in $\mathrm{XX}$ country and it is therefore crucial to avoid deterioration, reduce fear and support being active during that time. Signs of depression is a predictor for lower function and dissatisfaction after surgery for spinal stenosis $[30,31]$ and FABQ-PA is a predictor for worse outcome after surgery 
1 for disc herniation and spinal stenosis [32]. The level of FABQ-PA in this study was higher than

2 in a previous report for patients with acute and persistent LBP [33], but the same level as in

3 patients with spinal stenosis with a mean age of 75, where FABQ-PA > 16 was considered as

4 high [34]. This indicates that fear avoidance beliefs, depression and self-efficacy are areas of

5 interest to target in pre-surgery interventions.

6 At the 1-year follow-up in the current study, both groups improved in the PROMs, without

7 differences between the groups except for physical activity level. A larger proportion in the

8 physiotherapy-group had significantly higher physical activity level, which might have been

9 influenced by the pre-surgery physiotherapy. In a future 2-year follow-up, we will analyze if the

10 difference in physical activity level remains and if this influences recurrences of lumbar spine

11 disorders.

12 Our study is one of few studies evaluating pre-surgery intervention prior to spinal surgery.

13 Nielsen et al. [12] reported that 28 patients who received a 2 months daily exercise-program at

14 home pre-surgery, intense post-surgery physiotherapy together with optimized analgesic

treatment pre- and post-surgery improved in function pre-surgery and shortened the hospital-stay compared to a control-group. In our study, a supervised exercise-program was performed twice a week at a physiotherapy clinic to augment adherence, improve self-efficacy and reduce fear avoidance behaviour by providing feedback from a physiotherapist. Louw et al. [11] evaluated an intervention with neuroscience education compared to a control-group. The intervention-group reported that they were better prepared, that surgery had met their expectations to a higher extend, and they utilised less health care, both at the 1-year [11] and 3-year follow-up [35]. Considering these results, there seems to be of interest to further evaluate components targeting the patients understanding of expected outcomes, knowledge about pain-mechanism, exercises, being active and psychological factors. Compared to the current study both these two studies had more homogenous study populations including only patients with radicular pain $[11,12]$, while our study included also patients with pre-dominantly LBP. Furthermore, the previous study population had a mean duration of symptoms of just over 90 days and patients with other chronic 
1 pain syndromes, spinal stenosis and age $>65$ were excluded [11]. In our study, more than half of

2 the patients had LBP for $>2$ years and $35 \%$ leg pain for $>2$ years. Both long duration of

3 symptoms [36] and more LBP in relation to leg pain has shown to be predictors for inferior

4 outcome post-surgery in patients with disc herniation [37] and spinal stenosis [38].

5 Other reasons to lack of larger effects in function, pain and health in our study might be that the

6 pre-surgery intervention was not intense enough or may not have had enough treatment sessions

7 to improve health behavioural and the deconditioning, that a long duration of pain may cause.

8 Earlier implementation of our physiotherapy intervention might give better outcomes, as

9 recommended for LBP in general [39]. Notable is that only 115 patients (58\%) reported $\geq 1$

10 visits at a physiotherapist last 12 months and less than 50\% reported improvement. If patients

11 already had exhausted the non-surgical interventions or had experience only a few treatment-

12 sessions with physiotherapy with limited effect, that may have influenced their motivation and

13 expectation on outcomes of the pre-surgery physiotherapy.

14 Instead of pre-surgery intervention, physiotherapy has been compared to surgery in patients with spinal stenosis, showing similar effects at 2-year follow-up [40]. The physiotherapy included an exercise-program with conditioning exercises, tailor-made strengthening- and mobility-exercises, during 6 weeks at a physiotherapy clinic. Exercise-program and education is commonly the firstline intervention for patients with hip- and knee arthritis [41], and has also been suggested prior to arthroscopic subacromial decompression [42]. In similar way, an exercise-program might serve as a pre-surgery-selection for patients with degenerative lumbar disorders [40].

21 Considering predictors for outcome in spinal-surgery, many have recommended pre-surgery screening of psychosocial factors and use of psychosocially focused intervention [30, 32, 43]. In addition, recently, routine use of screening-tools for and treatment of depression during waitinglist time were recommended in a systematic review [44]. To our knowledge, these recommendations have not yet been studied. To reduce the risk for deteriorations during the waiting-list time and inferior outcome post-surgery in the heterogeneous-group, a pre-surgery selection could determine need of individualized additional treatment in conjunction to surgery. 
1 The pre-surgery intervention with education and home exercises $[11,12]$ have shown to be

2 sufficient for the homogenous groups studied. While a pre-surgery selection and tailor made

3 intervention with psychological support [44], analgesic treatments [12] and more specific

4 exercise programs as for spinal stenosis [40] and TBC might be useful for those with known risk

5 factors.

6 When interpreting our results, methodological strengths and limitations need to be considered.

7 The population awaiting surgery is heterogeneous and use of physiotherapy is suggested to be

8 based on tailored approaches. This is fulfilled by the use of TBC and tailoring the exercises for

9 each patients need. Eleven physiotherapy clinics delivered the pre-surgery physiotherapy, to

10 strengthen coherent administration between clinics, physiotherapists attended an introduction,

11 practice of the concept and had experience of treating these patients. Still some variations may

12 have appeared.

13 The current study used imputation and the sensitivity analysis supported use of the imputed 14 dataset a reasonable power was kept. The advantage of using imputed data is that all patients

15 included in the study remain in the analysis, even when some data are missing [27].

16 Our study is representative of XX context where patients are similar in terms of pre-surgery

17 PROMs to those registered in the national database XX [2]. Results of our study may also be 18 generalizable to other western world context.

19 The use of a MCIC [28] for the specific conditions of this study can be seen as a strength, but the 20 study specific analysis revealed smaller changes than those suggested in non-specific LBP and 21 post-surgical studies [24]. Smaller pre-surgery changes in this particular patient-group with 22 persistent pain might be of importance during the time on waiting-list.

\section{Conclusion}

The pre-surgery physiotherapy including TBC physiotherapy, tailor-made exercise-program and a behavioral approach decrease pain, risk for avoidance behavior and worsening of psychological 
spine disorder during the 2-3 months on waiting-list before surgery. There were no differences at

the 1-year post-surgery follow up, except for a higher activity level in the physiotherapy-group.

This might be beneficial to avoid recurrences in the long-term perspective. The pre-surgery

physiotherapy was well tolerated and without adverse events.

\section{References}

[1] Deyo RA, Mirza SK, Martin BI, Kreuter W, Goodman DC, Jarvik JG. Trends, major medical complications, and charges associated with surgery for lumbar spinal stenosis in older adults. Jama. 2010;303(13):1259-65.

[2] Reference excluded in this blinded mansucript. Available in a separate document in the submission.

[3] Machado GC, Witzleb AJ, Fritsch C, Maher CG, Ferreira PH, Ferreira ML. Patients with sciatica still experience pain and disability 5 years after surgery: A systematic review with meta-analysis of cohort studies. Eur J Pain. 2016.

[4] Fritsch CG, Ferreira ML, Maher CG, et al. The clinical course of pain and disability following surgery for spinal stenosis: a systematic review and meta-analysis of cohort studies. European spine journal : official publication of the European Spine Society, the European Spinal Deformity Society, and the European Section of the Cervical Spine Research Society. 2016.

[5] Kreiner DS, Shaffer WO, Baisden JL, et al. An evidence-based clinical guideline for the diagnosis and treatment of degenerative lumbar spinal stenosis (update). The spine journal : official journal of the North American Spine Society. 2013;13(7):734-43.

[6] Kreiner DS, Hwang SW, Easa JE, et al. An evidence-based clinical guideline for the diagnosis and treatment of lumbar disc herniation with radiculopathy. The spine journal : official journal of the North American Spine Society. 2014;14(1):180-91.

[7] Watters WC, 3rd, Bono CM, Gilbert TJ, et al. An evidence-based clinical guideline for the diagnosis and treatment of degenerative lumbar spondylolisthesis. The spine journal : official journal of the North American Spine Society. 2009;9(7):609-14.

[8] Group. EHTA. Treatment of degenerative lumbar spinal stenosis. Evidence report/technology assessment (Summary). 2001;(32):1-5.

[9] Carli F, Zavorsky GS. Optimizing functional exercise capacity in the elderly surgical population. Current opinion in clinical nutrition and metabolic care. 2005;8(1):23-32.

[10] Santa Mina D, Clarke H, Ritvo P, et al. Effect of total-body prehabilitation on postoperative outcomes: a systematic review and meta-analysis. Physiotherapy. 2014;100(3):196-207.

[11] Louw A, Diener I, Landers MR, Puentedura EJ. Preoperative pain neuroscience education for lumbar radiculopathy: a multicenter randomized controlled trial with 1-year follow-up. Spine. 2014;39(18):1449-57.

[12] Nielsen PR, Jorgensen LD, Dahl B, Pedersen T, Tonnesen H. Prehabilitation and early rehabilitation after spinal surgery: randomized clinical trial. Clinical rehabilitation. 2010;24(2):137-48.

[13] Stanton TR, Fritz JM, Hancock MJ, et al. Evaluation of a treatment-based classification algorithm for low back pain: a cross-sectional study. Physical therapy. 2011;91(4):496-509.

[14] Gibson JN, Waddell G. Surgical interventions for lumbar disc prolapse: updated Cochrane Review. Spine. 2007;32(16):1735-47.

[15] Reference to our study protocol, so excluded in this blinded mansucript. Available in a separate document in the submission. 
[16] Fairbank JC, Couper J, Davies JB, O'Brien JP. The Oswestry low back pain disability questionnaire. Physiotherapy. 1980;66(8):271-3.

[17] Scott J, Huskisson EC. Graphic representation of pain. Pain. 1976;2(2):175-84.

[18] Aaronson NK, Acquadro C, Alonso J, et al. International Quality of Life Assessment (IQOLA) Project. Qual Life Res. 1992;1(5):349-51.

[19] EuroQolGroup. EuroQol--a new facility for the measurement of health-related quality of life. Health Policy. 1990;16(3):199-208.

[20] Zigmond AS, Snaith RP. The hospital anxiety and depression scale. Acta psychiatrica Scandinavica. 1983;67(6):361-70.

[21] Altmaier E. Role of self-efficacy in rehabilitation outcome among chronic low back pain patients. Journal of Counseling Psychology. 1993;40(3):335-9.

[22] Waddell G, Newton M, Henderson I, Somerville D, Main CJ. A Fear-Avoidance Beliefs Questionnaire (FABQ) and the role of fear-avoidance beliefs in chronic low back pain and disability. Pain. 1993;52(2):157-68.

[23] Dworkin RH, Turk DC, Farrar JT, et al. Core outcome measures for chronic pain clinical trials: IMMPACT recommendations. Pain. 2005;113(1-2):9-19.

[24] Hagg O, Fritzell P, Nordwall A. The clinical importance of changes in outcome scores after treatment for chronic low back pain. European spine journal : official publication of the European Spine Society, the European Spinal Deformity Society, and the European Section of the Cervical Spine Research Society. 2003;12(1):12-20.

[25] Cohen J. A power primer. Psychological bulletin. 1992;112(1):155-9.

[26] Azur MJ, Stuart EA, Frangakis C, Leaf PJ. Multiple imputation by chained equations: what is it and how does it work? International journal of methods in psychiatric research. 2011;20(1):40-9.

[27] Sterne JA, White IR, Carlin JB, et al. Multiple imputation for missing data in epidemiological and clinical research: potential and pitfalls. BMJ (Clinical research ed). 2009;338:b2393.

[28] van der Roer N, Ostelo RW, Bekkering GE, van Tulder MW, de Vet HC. Minimal clinically important change for pain intensity, functional status, and general health status in patients with nonspecific low back pain. Spine. 2006;31(5):578-82.

[29] Soer R, Reneman MF, Vroomen PC, Stegeman P, Coppes MH. Responsiveness and minimal clinically important change of the Pain Disability Index in patients with chronic back pain. Spine. 2012;37(8):711-5.

[30] Sinikallio S, Lehto SM, Aalto T, Airaksinen O, Kroger H, Viinamaki H. Depressive symptoms during rehabilitation period predict poor outcome of lumbar spinal stenosis surgery: a two-year perspective. BMC musculoskeletal disorders. 2010;11:152.

[31] Adogwa O, Parker SL, Shau DN, et al. Preoperative Zung depression scale predicts patient satisfaction independent of the extent of improvement after revision lumbar surgery. The spine journal : official journal of the North American Spine Society. 2013;13(5):501-6.

[32] Havakeshian S, Mannion AF. Negative beliefs and psychological disturbance in spine surgery patients: a cause or consequence of a poor treatment outcome? European spine journal : official publication of the European Spine Society, the European Spinal Deformity Society, and the European Section of the Cervical Spine Research Society. 2013;22(12):2827-35.

[33] Grotle M, Vollestad NK, Brox JI. Clinical course and impact of fear-avoidance beliefs in low back pain: prospective cohort study of acute and chronic low back pain: II. Spine. 2006;31(9):1038-46.

[34] Burgstaller JM, Wertli MM, Steurer J, Kessels AG, Held U, Gramke HF. The Influence of Pre- and Postoperative Fear Avoidance Beliefs on Postoperative Pain and Disability in Patients With Lumbar Spinal Stenosis: Analysis of the Lumbar Spinal Outcome Study (LSOS) Data. Spine. 2017;42(7):E425-e32.

[35] Louw A, Diener I, Landers MR, Zimney K, Puentedura EJ. Three-year follow-up of a randomized controlled trial comparing preoperative neuroscience education for patients 
undergoing surgery for lumbar radiculopathy. Journal of spine surgery (Hong Kong). 2016;2(4):289-98.

[36] Wilson CA, Roffey DM, Chow D, Alkherayf F, Wai EK. A systematic review of preoperative predictors for postoperative clinical outcomes following lumbar discectomy. The spine journal : official journal of the North American Spine Society. 2016;16(11):141322.

[37] Kleinstueck FS, Fekete T, Jeszenszky D, et al. The outcome of decompression surgery for lumbar herniated disc is influenced by the level of concomitant preoperative low back pain. European spine journal : official publication of the European Spine Society, the European Spinal Deformity Society, and the European Section of the Cervical Spine Research Society. 2011;20(7):1166-73.

[38] Kleinstuck FS, Grob D, Lattig F, et al. The influence of preoperative back pain on the outcome of lumbar decompression surgery. Spine. 2009;34(11):1198-203.

[39] Koes BW, van Tulder M, Lin CW, Macedo LG, McAuley J, Maher C. An updated overview of clinical guidelines for the management of non-specific low back pain in primary care. European spine journal : official publication of the European Spine Society, the European Spinal Deformity Society, and the European Section of the Cervical Spine Research Society. 2010;19(12):2075-94.

[40] Delitto A, Piva SR, Moore CG, et al. Surgery versus nonsurgical treatment of lumbar spinal stenosis: a randomized trial. Ann Intern Med. 2015;162(7):465-73.

[41] Fernandes L, Hagen KB, Bijlsma JW, et al. EULAR recommendations for the nonpharmacological core management of hip and knee osteoarthritis. Annals of the rheumatic diseases. 2013;72(7):1125-35.

[42] Holmgren T, Bjornsson Hallgren H, Oberg B, Adolfsson L, Johansson K. Effect of specific exercise strategy on need for surgery in patients with subacromial impingement syndrome: randomised controlled study. BMJ (Clinical research ed). 2012;344:e787.

[43] Adogwa O, Carr K, Fatemi P, et al. Psychosocial factors and surgical outcomes: are elderly depressed patients less satisfied with surgery? Spine. 2014;39(19):1614-9.

[44] Dorow M, Lobner M, Stein J, et al. Risk Factors for Postoperative Pain Intensity in Patients Undergoing Lumbar Disc Surgery: A Systematic Review. PloS one. 2017;12(1):e0170303. 


\section{Figure Captions}

2 Fig. 1. CONSORT flow chart of the randomized controlled trial.

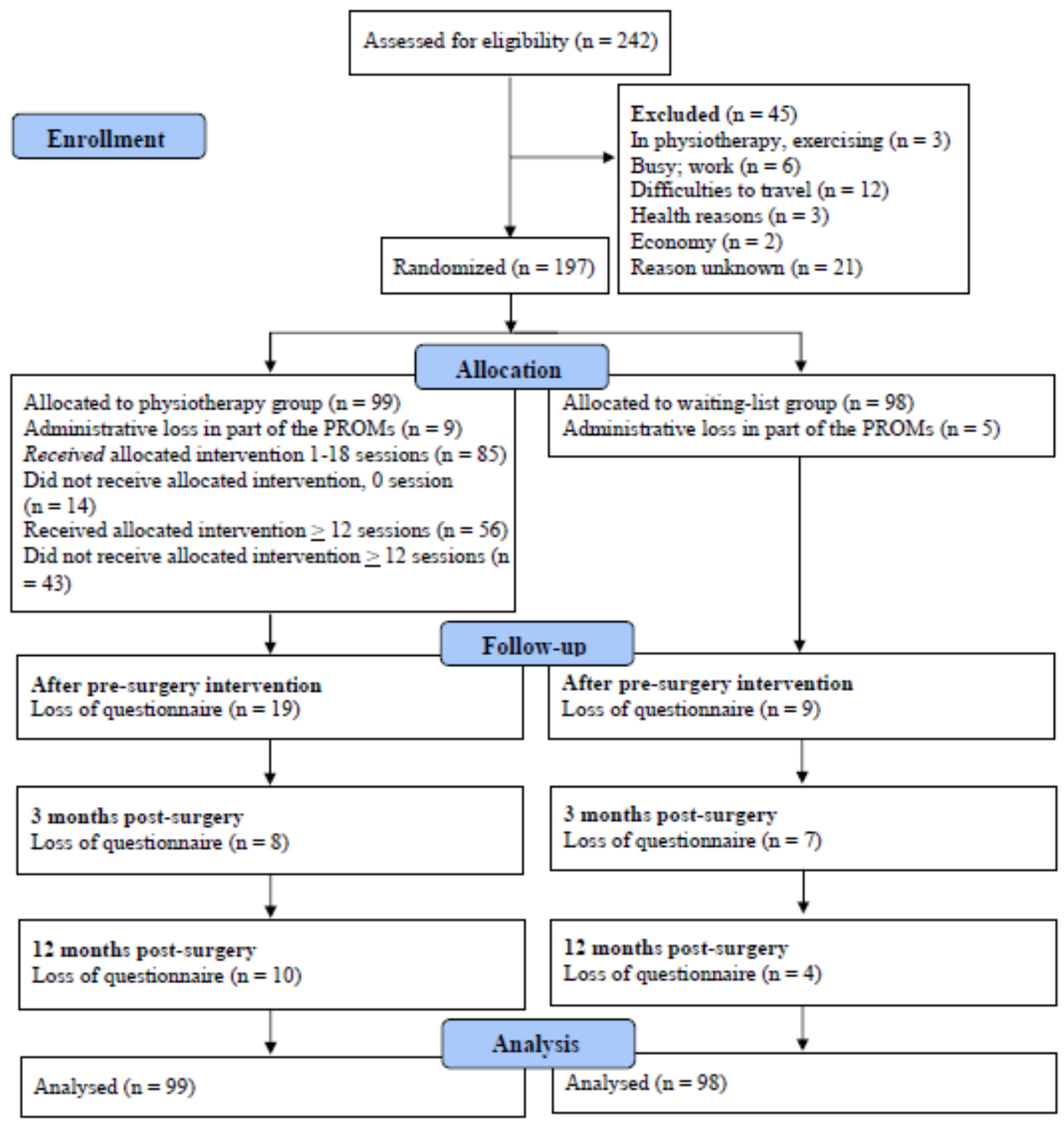

4 Fig. 2. Patient Global Impression of Change after pre-surgery intervention for the physiotherapy-

$5 \quad(\mathrm{n}=99)$ and waiting-list group $(\mathrm{n}=98)$. Original data. 


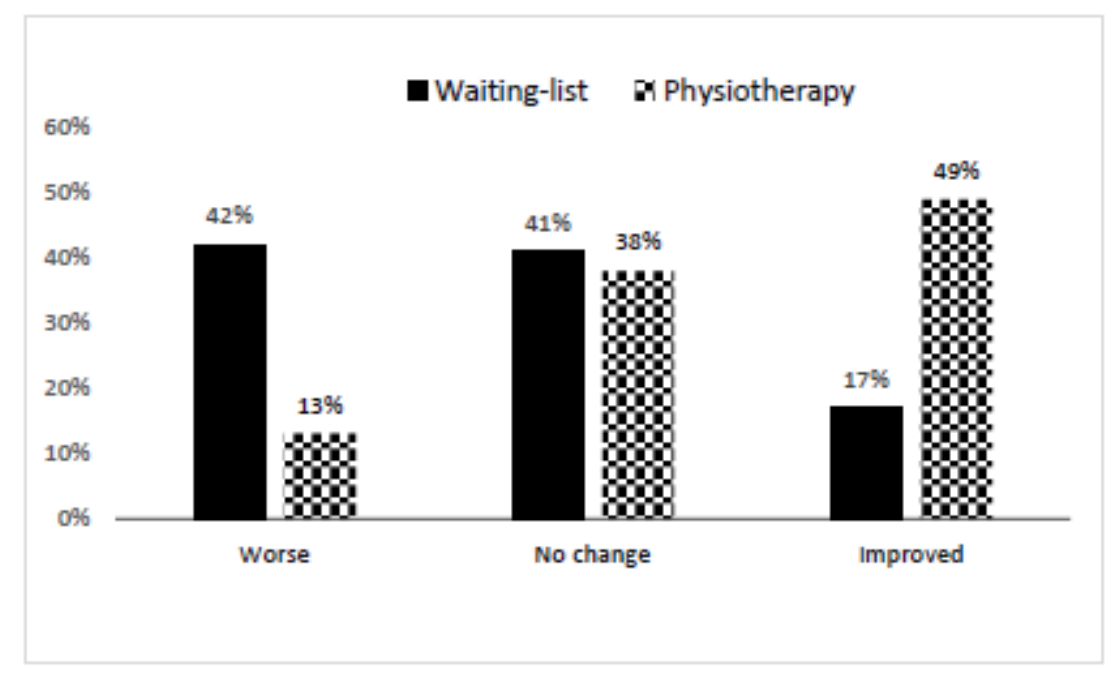

1

Table 1 Baseline characteristics for patients with degenerative lumbar disorder scheduled for surgery. Comparison between patients allocated to the physiotherapy- and the waiting-list group.

\begin{tabular}{|c|c|c|c|}
\hline & Physiotherapy $(\mathrm{n}=99)$ & Waiting-list $(\mathrm{n}=98)$ & $P$-value \\
\hline Age, mean (SD) & $58(13.3)$ & $61(11.5)$ & 0.082 \\
\hline Gender, women $n(\%)$ & $54(54)$ & $51(52)$ & 0.725 \\
\hline Cigarette smoker, $n(\%)$ & $9(10)$ & $9(10)$ & 0.922 \\
\hline Prior spinal surgery, not for the same segment, $\mathrm{n}(\%)$ & & & 0.406 \\
\hline 1 & $12(13)$ & $7(8)$ & \\
\hline$\geq 2($ number of surgeries $2-5)$ & $7(8)$ & $6(6)$ & \\
\hline Employment situation, $\mathrm{n}(\%)$ & & & 0.232 \\
\hline Currently working & $37(38)$ & $27(28)$ & \\
\hline Unemployed & $2(2)$ & 0 & \\
\hline Retired & $37(38)$ & $45(47)$ & \\
\hline Sick leave and/or retired due to health problems & $19(19)$ & $24(24)$ & \\
\hline Physical activity last 12 months, n (\%) & & & 0.163 \\
\hline Inactive & $9(9.4)$ & $19(19,6)$ & \\
\hline Mildly active & $15(15.6)$ & $20(20.6)$ & \\
\hline
\end{tabular}


Walking

Moderately active

Very active

Diagnosis, n (\%)

Spinal stenosis

Disc herniation

Spondylolisthes

Degenerative Disc Disease

Pain duration back- /leg pain > 1-year, n (\%)
$48(50)$

$22(22.9)$

$2(2.1)$

$3(3.1)$
$40(41.2)$

$15(15.5)$

0.286

59 (60)

$70(71)$

$23(23)$

17 (17)

$8(8)$

7 (7)

$9(9)$

4 (4)

$57(64)$
$62(67)$

0.635 the waiting-list group.

\begin{tabular}{llllll}
\hline & \multicolumn{4}{l}{ Physiotherapy $(\mathrm{n}=99)$} & \multicolumn{2}{l}{ Waiting-list $(\mathrm{n}=98)$} \\
\hline & Mean & $(\mathrm{SD})$ & Mean & $(\mathrm{SD})$ & P-value \\
\hline ODI & 37.9 & $(12.8)$ & 40.5 & $(12.6)$ & 0.155 \\
VAS back pain & 56.0 & $(24.4)$ & 59.7 & $(21.6)$ & 0.264 \\
VAS leg pain & 65.3 & $(22.1)$ & 64.0 & $(20.0)$ & 0.670 \\
EQ-5D & 0.371 & $(0.3)$ & 0.356 & $(0.3)$ & 0.720 \\
EQVAS & 50.7 & $(18.3)$ & 47.7 & $(20.5)$ & 0.280 \\
SF-36 PCS & 29.7 & $(6.7)$ & 27.2 & $(7.2)$ & $\mathbf{0 . 0 1 2}$ \\
SF-36 MCS & 45.2 & $(12.9)$ & 44.0 & $(13.1)$ & 0.520 \\
FABQ-PA & 16.0 & $(5.8)$ & 16.0 & $(5.2)$ & 0.993 \\
HADS anxiety & 5.4 & $(4.0)$ & 5.6 & $(3.8)$ & 0.668 \\
HADS depression & 4.4 & $(3.5)$ & 4.4 & $(3.0)$ & 0.955 \\
SES & 134 & $(38.3)$ & 127 & $(30.7)$ & 0.142
\end{tabular}

Bold text $\mathbf{p}<\mathbf{0 . 0 5} \mathrm{SD}$, standard deviation.

3 Table 2 Mean (SD) of patient reported outcome measures at baseline for patients allocated to the physiotherapy-and

\section{Bold text $\mathrm{p}<0.05$}

6 SD, Standard Deviation; ODI, Oswestry Disability Index (0-100) (higher score indicates higher disability); VAS,

7 Visual Analog Scale (0-100) (higher score indicates higher pain intensity); EQ-5D, EuroQol (-0.594 to 1) (higher

8 score indicates better health); SF-36 PCS and MCS, Short Form-36 Health related quality of life Physical and

9 Mental health Component Summary scores (0-100) (higher score indicate better health); FABQ-PA, Fear-Avoidance 
1 Beliefs Physical Activity (0-24) (higher score indicates more signs of fear-avoidance); HADS, Hospital Anxiety and

2 Depression Scale (0-21) (higher score indicates more signs of symptoms); SES, Self-Efficacy Scale (0-200) (higher

3 score indicates better self-efficacy). Data with imputation.

4

5 Table 3. Within and between group differences over time in patient reported outcome measures between baseline and after pre-

6 surgery intervention, 3 months and 1-year post-surgery.

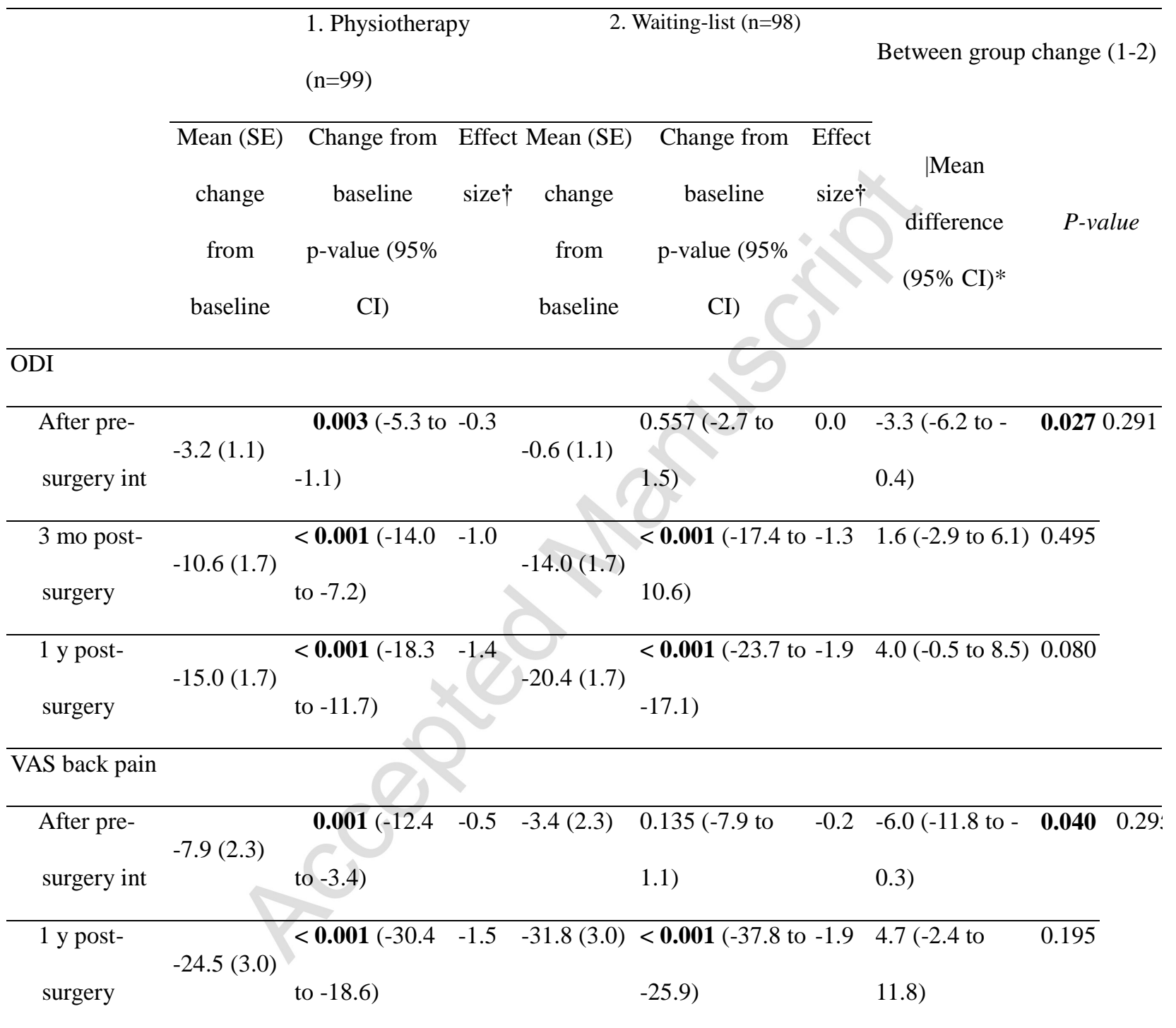

VAS leg pain

\begin{tabular}{|c|c|c|c|c|c|c|c|}
\hline After pre- & $-10.5(2.5)$ & $<0.001(-15.4$ & -0.5 & $-5.0(2.5)$ & $\mathbf{0 . 0 4 4}(-9.8$ to $-\quad-0.2$ & $-4.6(-10.8$ to & 0.140 \\
\hline surgery int & & to -5.5$)$ & & & $0.1)$ & 1.5) & \\
\hline 1 y post- & $-35.0(3.3)$ & $<0.001(-41.5$ & -1.4 & $-36.5(3.3)$ & $<\mathbf{0 . 0 0 1}(-43.0$ to -1.4 & $2.9(-5.4$ to & 0.484 \\
\hline surgery & & to -28.5$)$ & & & $-30.0)$ & 11.2) & \\
\hline
\end{tabular}

EQ-5D

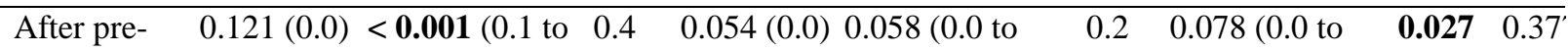




\begin{tabular}{|c|c|c|c|c|c|c|c|}
\hline surgery int & & $0.2)$ & & $0.1)$ & $0.1)$ & & \\
\hline 3 mo post- & $0.267(0.0)$ & $<\mathbf{0 . 0 0 1}(0.2$ to 1.0 & $0.281(0.0)$ & $<\mathbf{0 . 0 0 1}(0.2$ to 1.0 & $0.004(-0.1$ to & 0.902 & \\
\hline surgery & & $0.3)$ & & $0.3)$ & $0.1)$ & & \\
\hline 1 y post- & $0.280(0.0)$ & $<\mathbf{0 . 0 0 1}(0.2$ to 1.0 & $0.329(0.0)$ & $<\mathbf{0 . 0 0 1}(0.3$ to 1.2 & $-0.030(-0.1$ to & 0.455 & \\
\hline surgery & & $0.4)$ & & $0.4)$ & $0.0)$ & & \\
\hline \multicolumn{8}{|l|}{ EQ-VAS } \\
\hline After pre- & $5.4(1.6)$ & $\mathbf{0 . 0 0 1}$ (2.3 to 0.3 & $1.8(1.6)$ & $0.253(-1.3$ to & $4.6(0.4$ to 8.8$)$ & 0.031 & $\overline{0.31}$ \\
\hline surgery int & & $8.6)$ & & $5.0)$ & & & \\
\hline 3 mo post- & $18.3(2.2)$ & $<\mathbf{0 . 0 0 1}(13.9$ & $19.6(2.3)$ & $<\mathbf{0 . 0 0 1}$ (15.2 to 1.0 & $0.8(-4.5$ to 6.1$)$ & 0.769 & \\
\hline surgery & & to 22.8 ) & & 24.1) & & & \\
\hline 1 y post- & $16.8(2.4)$ & $\begin{array}{l}<\mathbf{0 . 0 0 1}(12.1 \quad 0.8 \\
\end{array}$ & $23.5(2.4)$ & $<\mathbf{0 . 0 0 1}$ (18.7 to 1.2 & $-4.5(-10.3$ to & 0.121 & \\
\hline surgery & & to 21.5 ) & & 28.2) & 1.2) & & \\
\hline \multicolumn{8}{|l|}{ SF-36 PCS } \\
\hline After pre- & $2.4(0.7)$ & $\mathbf{0 . 0 0 1}$ (0.9 to 0.3 & $1.6(0.7)$ & $\mathbf{0 . 0 2 8}(0.2$ to & $-1.5(-0.5$ to & 0.129 & 0.07 . \\
\hline surgery int & & 3.8) & & $3.1)$ & $3.5)$ & & \\
\hline 1 y post- & $10.0(1.2)$ & $\begin{array}{l}\mathbf{0 . 0 0 1}(-12.4 \quad 1.0 \\
\end{array}$ & $12.5(1.3)$ & $<\mathbf{0 . 0 0 1}$ (10.1 to 1.2 & $-1.4(-4.8$ to & 0.404 & \\
\hline surgery & & to -7.6$)$ & & 14.9) & 1.9) & & \\
\hline
\end{tabular}

\section{SF-36 MCS}

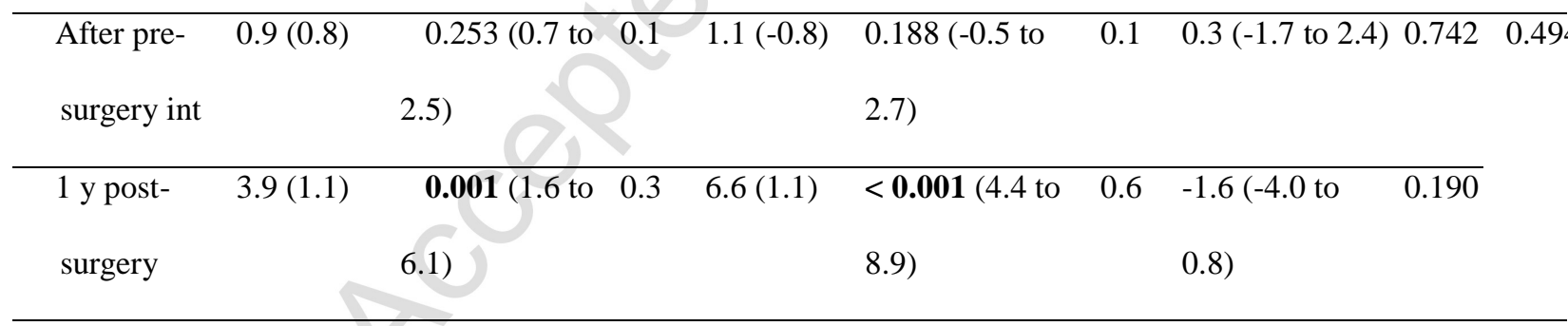

\section{FABQ-PA}

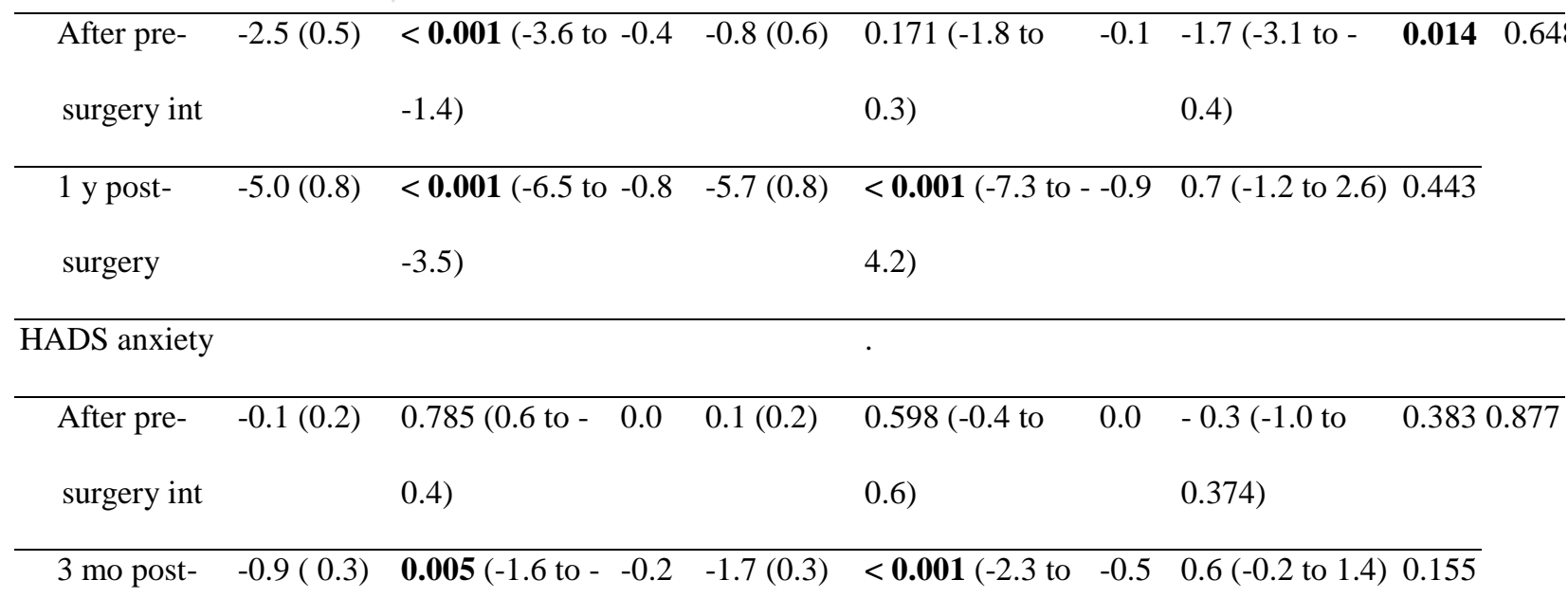




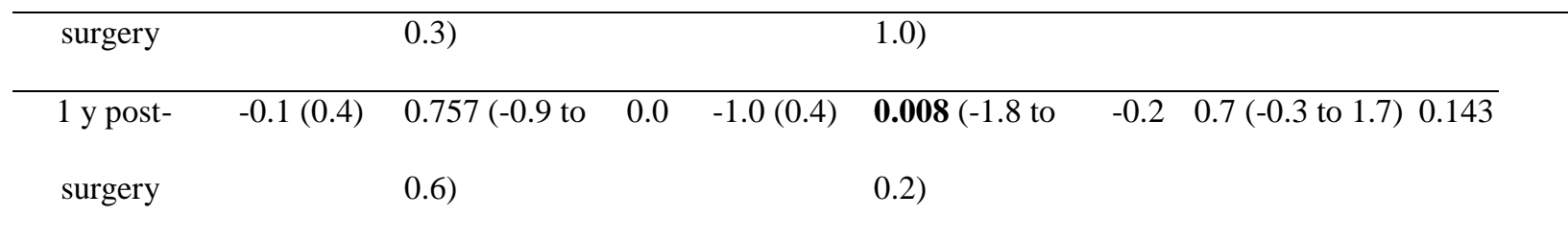

\section{HADS}

depression

\begin{tabular}{|c|c|c|c|c|c|c|c|c|}
\hline $\begin{array}{l}\text { After pre- } \\
\text { surgery int }\end{array}$ & $0.0(0.2)$ & $\begin{array}{l}0.975(0.5 \text { to }- \\
0.5)\end{array}$ & 0.0 & $1.0(0.2)$ & $\begin{array}{l}<\mathbf{0 . 0 0 1} \wedge(0.6 \text { to } \\
1.5)\end{array}$ & $0.3^{\wedge}$ & $\begin{array}{l}-1.0(-1.7 \text { to }- \\
0.4)\end{array}$ & $\mathbf{0 . 0 0 2} 0.539$ \\
\hline 3 mo post- & $-0.9(0.3)$ & $\mathbf{0 . 0 0 2}(-1.5$ to & -0.3 & $-1.0(0.3)$ & $\mathbf{0 . 0 0 1}(-1.6$ to & -0.3 & $0.064(-0.7$ to & 0.863 \\
\hline surgery & & $0.3)$ & & & $0.4)$ & & $0.8)$ & \\
\hline 1 y post- & $-0.5(0.4)$ & $0.208(-1.2$ to & -0.1 & $-0.6(0.4)$ & $0.108(-1.3$ to & -0.2 & $0.1(-0.8$ to 1.0$)$ & 0.809 \\
\hline surgery & & $0.2)$ & & & $0.1)$ & & & \\
\hline
\end{tabular}

\section{SES}

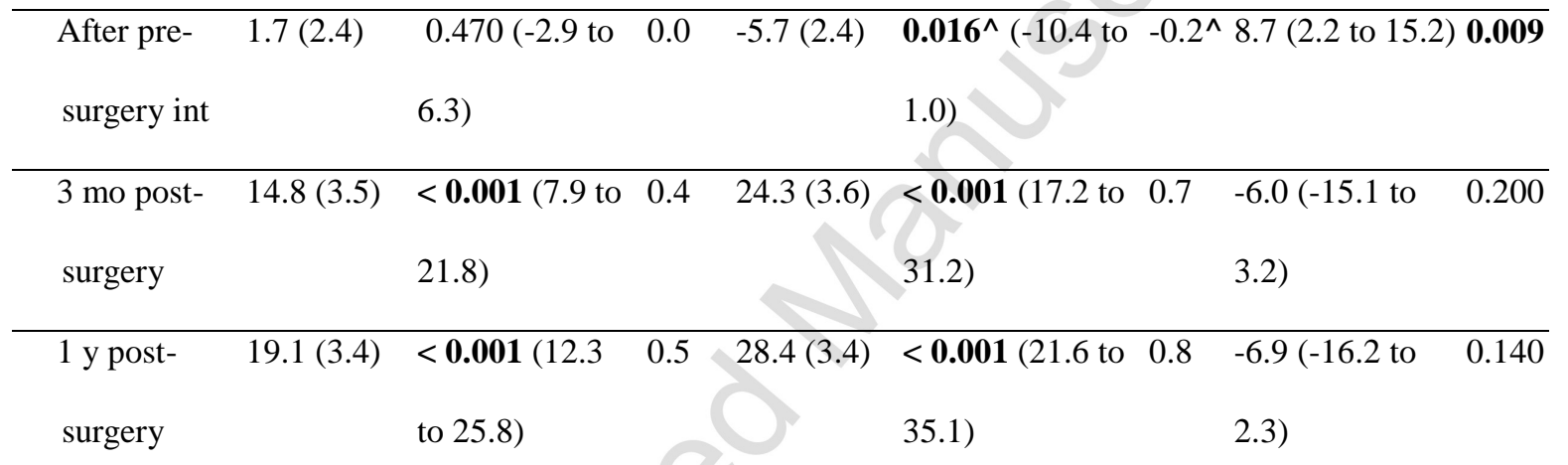

$\overline{\text { Age, gender and diagnosis (disc herniation and degenerative disc disease / spinal stenosis and spondylolistheses) was }}$ used as covariates * Adjusted for score at baseline, $\dagger$ Cohen's d, ^ detoriation Bold text $\mathbf{p}<\mathbf{0 . 0 5}$ int Intervention; SD, Standard Deviation; CI, confidence interval, ODI, Oswestry Disability Index (0-100) (higher score indicates higher disability); VAS, Visual Analog Scale (0-100) (higher score indicates higher pain intensity); EQ-5D, EuroQol (-0.594 to 1) (higher score indicates better health); SF-36 PCS and MCS, Short Form-36 Health related quality of life Physical and Mental health Component Summary scores (0-100) (higher score indicate better health); FABQ-PA, Fear-Avoidance Beliefs Physical Activity (0-24) (higher score indicates more signs of fearavoidance); HADS, Hospital Anxiety and Depression Scale (0-21) (higher score indicates more signs of symptoms); SES, Self-Efficacy Scale (0-200) (higher score indicates better self-efficacy). Data with imputation.

11 Table 4 The significant mean changes in PROMs after the pre-surgery intervention in comparison to minimal 12 detectible change and optimal cutoff point of minimal clinical important change.

\section{Physiotherapy $(\mathrm{n}=99)$}




\begin{tabular}{lllllll}
\hline & \multicolumn{2}{l}{ Mean $(\mathrm{SE})$ change from baseline } & MDC & OCP & $($ Sens; Spec $)$ & AUC $(P$-value $)$ \\
\hline ODI & 3.2 & $(1.1)$ & 2.9 & 3.3 & $(0.62 ; 0.70)$ & $0.72(<0.001)$ \\
VAS back & 7.9 & $(2.3)$ & 6.3 & 7.0 & $(0.70 ; 0.56)$ & $0.68(<0.001)$ \\
VAS leg & 10.5 & $(2.5)$ & 6.8 & 7.8 & $(0.72 ; 0.64)$ & $0.75(<0.001)$ \\
EQ-5D & -0.121 & $0.0)$ & 0.08 & 0.07 & $(0.62 ; 0.55)$ & $0.59(0.066)$ \\
EQ-VAS & -5.5 & $(1.6)$ & 4.4 & 4.5 & $(0.64 ; 0.52)$ & $0.65(<0.001)$ \\
SF-36 PCS & 2.4 & $(0.7)$ & 2.0 & 2.7 & $(0.70 ; 0.68)$ & $0.76(<0.001)$ \\
FABQ-PA & 2.5 & $(0.5)$ & 1.5 & 1.9 & $(0.65 ; 0.61)$ & $0.70(<0.001)$ \\
\hline & Waiting-list (n=98) & & & & & \\
\hline
\end{tabular}

1 SE, Standard Error; MDC, Minimal Detectable Change; OCP, Optimal Cutoff Point; Sens, sensitivity; Spec,

2 specificity; AUC, Area Under the Curve.

3 